\title{
A Novel Software Tool for Detection of Meniscus Injury using Dynamic Fuzzy Cognitive Networks
}

\author{
Anninou AP ${ }^{1 *}$, Poulios $\mathbf{P}^{2}$, Groumpos $\mathbf{P P}^{1}$ and Gliatis $\mathbf{I}^{2}$ \\ ${ }^{1}$ Department of Electrical and Computer Engineering, Laboratory of Automation and Robotics, University of Patras, Greece \\ ${ }^{2}$ Department of Medicine, University of Patras, Greece
}

\begin{abstract}
Objective: The main objective of this study is the construction of a tool to diagnose a specific type of knee injuries, the meniscus injury, without the aid of imaging modalities. This tool exploits physician's knowledge and experience and extracts all information needed for a diagnostic procedure.

Methods: A specific type of Fuzzy Cognitive Maps, which is named Dynamic Fuzzy Cognitive Networks, is used for the implementation of diagnostic process. This method simulates the physician's way of thinking and making decisions in order to conclude to a final diagnosis and will constitute the background of a user interface platform, named "KneeD," designed especially as a supportive tool for the physician. It exploits information about patient history and clinical examination and the arisen symptoms and risk factors constitute the main attributes that contribute to injury identification.

Results: A pilot sample of 17 patients with knee injuries who arrived at the University Hospital of Patras, in Greece, was examined by the orthopedic clinician and the diagnoses confirmed with MRI were compared to our platform's outcomes. Results concerned a) the initial distinction between "meniscus injury" and "other disease" and b) discrimination between acute and degenerative injury for patients positive to meniscal tear from the first level. Both levels provided us with very satisfactory results in total agreement with MRI outcomes.

Conclusion: The outcome of a close collaboration between engineers and medical doctors was a platform for real time diagnosing of knee injuries, which is simple, user friendly, real-time, easily accessible, fast, reliable, and low-cost. Furthermore, it could also be used as a patient storage database. The obtained results have been evaluated by orthopedic surgeons who found them very satisfactory.
\end{abstract}

Keywords: Clinical diagnosis; Knee injuries; Meniscus; Dynamic Fuzzy Cognitive Networks; Decision making; Platform; Database

\section{Introduction}

Soft tissue knee injuries are among the most common clinical challenges in the Emergency Department [1-3]. An acute knee injury after a sport accident could be the cause of a permanent disability [4]. Initial management of these injuries is critical. Non orthopedic medical staff from Emergency department usually suggests rest at home or a direct referral to the orthopedic department. The last one requires assessment of the condition of the patient and review by orthopedic surgeons. This, however leads to multiple visits to the clinic and is often ineffective since the surgeon should review all injuries and only a small percentage of them require special intervention [5]. Therefore, a selection process is required so that patients receive the correct management direction. Accurate and timely diagnosis increases the chance of complete recovery of the affected knee.

Meniscus tear is a well-known knee disorder and constitutes a risk factor for the subsequent development and progression of osteoarthritis or even partial disability.

Magnetic resonance imaging (MRI) is of the utmost importance for an accurate, non-invasive assessment of internal knee pathology [6]. The use of MRI has been increased over the last years, reducing the emphasis given by physicians on physical examination. The necessity of MRI for evaluating knee injuries has been called into question because of the high cost and the proven number of false positive results especially regarding the medial meniscus pathology $[7,8]$. Nevertheless, their number continues to increase. It is up to the physicians to prove that diagnostic studies are both beneficial and cost-effective [9].
A detailed history of patient and a comprehensive physical examination are still considered as the cornerstones of the diagnosis of meniscal injuries. However, the results of physical examination are usually quite ambiguous or doubtful about the complex or even the simplest patterns of soft tissue knee injuries, resulting in a variety of conflicting findings. That perception creates the necessity of a completely different perspective for dealing with the problem.

Taking into consideration all these aforementioned reasons, we have tried to build a well-structured and systematic tool, including both physical examination and a kind of "interview" (questions-answers) to the patient in order to accurately assess and localize the injury [10]. But there are too many and complex parameters to be taken into account for an appropriate decision. In this tool the orthopedic clinician plays a crucial role. Fuzzy Cognitive Maps (FCM) methodology could solve the problems by extracting knowledge and experience and by simulating physician's diagnostic process. This implementation has two levels:

*Corresponding author: Antigoni P. Anninou, Department of Electrical and Computer Engineering, Laboratory of Automation and Robotics, University of Patras, 26504, Patras, Greece, Tel: +302610996450; E-mail: anninou@ece.upatras.gr

Received: January 30, 2018; Accepted: March 06, 2018; Published: March 12 2018

Citation: Anninou AP, Poulios P, Groumpos PP, Gliatis I (2018) A Novel Software Tool for Detection of Meniscus Injury using Dynamic Fuzzy Cognitive Networks. Physiother Rehabil 3: 155. doi: 10.4172/2573-0312.1000155

Copyright: (c) 2018 Anninou AP, et al. This is an open-access article distributed under the terms of the Creative Commons Attribution License, which permits unrestricted use, distribution, and reproduction in any medium, provided the original author and source are credited. 
1) Meniscus Injury Diagnosis

2) Acute or degenerative injury diagnosis

In the first level the two possible diagnoses are "Meniscus" and "Other Disease". If the patient is positive to meniscus then we move to the next level for an accurate diagnosis, between acute and degenerative option. Method is thoroughly analyzed in [10].

Dynamic Fuzzy Cognitive Networks (DFCN), which will be presented in the next section, will model the disease. The whole platform is presented in section 3. Section 4 includes the results from 17 patients tested. Paper ends up with conclusions and future research.

\section{Dynamic fuzzy cognitive networks}

FCM is a representation of knowledge. It is a fuzzy-graph structure for representing causal reasoning. Its fuzziness allows hazy degrees of causality between hazy causal objects (concepts) [11,12]. It is a promising modeling method for describing particular domains showing the concepts (variables) and the relationships between them (weights) while it encompasses advantageous features. FCM was firstly introduced by Kosko in 1986. It's a 30 years old methodology that has been tested through a wide range of applications in many fields and is continuously evolved in order to overcome the disadvantages. Due to the drawbacks of the classic methodology, a new evolutionary model was proposed by the authors in [10]. This method, Dynamic Fuzzy Cognitive Networks (DFCN), consists of three kinds of variables: Fuzzy States, Fuzzy Inputs and Fuzzy Outputs and two equations:

$$
\begin{aligned}
& \mathbf{x}(\mathrm{k}+1)=\mathrm{f}[\mathrm{Ax}(\mathrm{k})+\mathrm{Bu}(\mathrm{k})] \\
& \mathbf{y}(\mathrm{k})=\mathrm{f}[\mathrm{Cx}(\mathrm{k})+\mathrm{Du}(\mathrm{k})]
\end{aligned}
$$

where $u(k) € R^{r}$ is an exogenous stimulation input vector, $x(k) € R^{n}$ is a state vector, $y(k) € R^{m}$ is the output vector in time variable " $k$ ". Nonlinear function $f(\cdot)$ can be approximated using a nonlinear activation function, like sigmoid function. Array A is a weight matrix that connects the states between them. Array B is a weight matrix which represents how each input influences the states one by one. Array $\mathrm{C}$ contains the interrelationships between states and outputs and array $\mathrm{D}$ the influence of the inputs to the outputs respectively. These arrays must be decided by experts. The exact procedure is the same as in the construction of a classic Fuzzy Cognitive Map [12-14]. Experts should define all these interconnections in their natural language using linguistic variables and after a defuzzification method the arrays will be filled with numeric values [13].

In order to implement the above methodology into meniscus injury diagnosis we should first categorize the main variables of the disease to each vector of Eq. (1) and Eq. (2). In the first level we have the following concepts:

Outputs: Meniscus-Other Disease

States: 13 Symptoms (Table 1)

Inputs: No

In the second level:

Outputs: Acute Injury-Degenerative Injury

States: 17 Risk Factors (Table 2)

\section{Inputs: No}

The patient symptoms are relied on a questionnaire with expanded

\begin{tabular}{|c|c|}
\hline Checklist & Expanded Description \\
\hline C1: Clicking & $\begin{array}{c}\text { Do you feel a clicking sensation or hear a clicking } \\
\text { noise when you move your knee? }\end{array}$ \\
\hline C2: Catching & $\begin{array}{l}\text { Do you feel that sometimes something is caught in } \\
\text { your knee that momentarily prevents movement? }\end{array}$ \\
\hline $\begin{array}{l}\text { C3: Giving way/ } \\
\text { weakness }\end{array}$ & $\begin{array}{l}\text { Do you sometimes feel that your knee will give out and } \\
\text { not support your weight? }\end{array}$ \\
\hline C4: Localized pain & $\begin{array}{l}\text { Is your knee pain centered to one spot on the knee } \\
\text { that you can point to with your finger? }\end{array}$ \\
\hline C5: Episodic pain & $\begin{array}{l}\text { Do you have pain that comes and goes with specific } \\
\text { movements and activities? }\end{array}$ \\
\hline C6: Pain with activity & $\begin{array}{l}\text { Do you experience pain that is caused by specific } \\
\text { activities? }\end{array}$ \\
\hline $\begin{array}{l}\text { C7: Pain with pivoting/ } \\
\text { twisting }\end{array}$ & Do you feel pain when you pivot or twist your knee? \\
\hline $\begin{array}{l}\text { C8: Change in quality/ } \\
\text { pattern of pain }\end{array}$ & $\begin{array}{l}\text { Have you had a change in type, location, or frequency } \\
\text { of your pain? }\end{array}$ \\
\hline C9: Locking & $\begin{array}{l}\text { Do you feel that your knee sometimes gets stuck } \\
\text { temporarily so that you can't move it further? }\end{array}$ \\
\hline C10: Acute swelling & $\begin{array}{l}\text { Did your knee get swollen immediately or in } 2 \text { hours } \\
\text { after injury? }\end{array}$ \\
\hline C11: Subacute swelling & $\begin{array}{l}\text { Did your knee get swollen gradually after the injury up } \\
\text { to the next day? }\end{array}$ \\
\hline C12: Weight bearing & $\begin{array}{l}\text { Was it possible for you to stand up and walk after } \\
\text { injury? }\end{array}$ \\
\hline $\begin{array}{l}\text { C13: Continued in } \\
\text { athletic activity }\end{array}$ & Did you continue to your activity after the injury? \\
\hline
\end{tabular}
descriptions of the checklist of meniscal symptoms as in Table 1 .
Table 1: Symptoms Checklist.

\begin{tabular}{|c|c|}
\hline R1 & Sport activity/high Tegner score (soccer) \\
\hline R2 & Systemic laxity $(>1$ score in Beighton scale) \\
\hline R3 & History of previous injury/surgery \\
\hline R4 & Time From initial ACL Injury $>2-5$ years \\
\hline R5 & Lifting weight routine \\
\hline R6 & Age $>60$ years old \\
\hline R7 & Male gender \\
\hline R8 & Work related/frequently kneeling-squatting \\
\hline R9 & Using stairs frequently \\
\hline R10 & BMl_25 kgr/m ${ }^{2}$ \\
\hline R11 & Standing/walking routine \\
\hline R12 & Chronic recurring pain and swelling after exercise \\
\hline R13 & Minor trauma/lnsignificant traumatic injury/unable to recall traumatic \\
\hline event
\end{tabular}

Table 2: Risk factors.

The patients are also assessed for risk factors which are attributes, characteristics or exposures that increase the likelihood of a person developing a disease, in order to check if they are susceptible to acute or degenerative meniscal tears respectively. The list of 17 risk factors is in Table 2.

We use the new equations in two different subsystems. Both the procedure and the points where DFCN is applied are depicted in Figure 1.

\section{KneeD Platform}

The step by step procedure is the following:

Step 1: Physicians define all basic concepts that influence meniscus tear.

Step 2: Engineers separate them into Fuzzy States, Inputs and Outputs. 


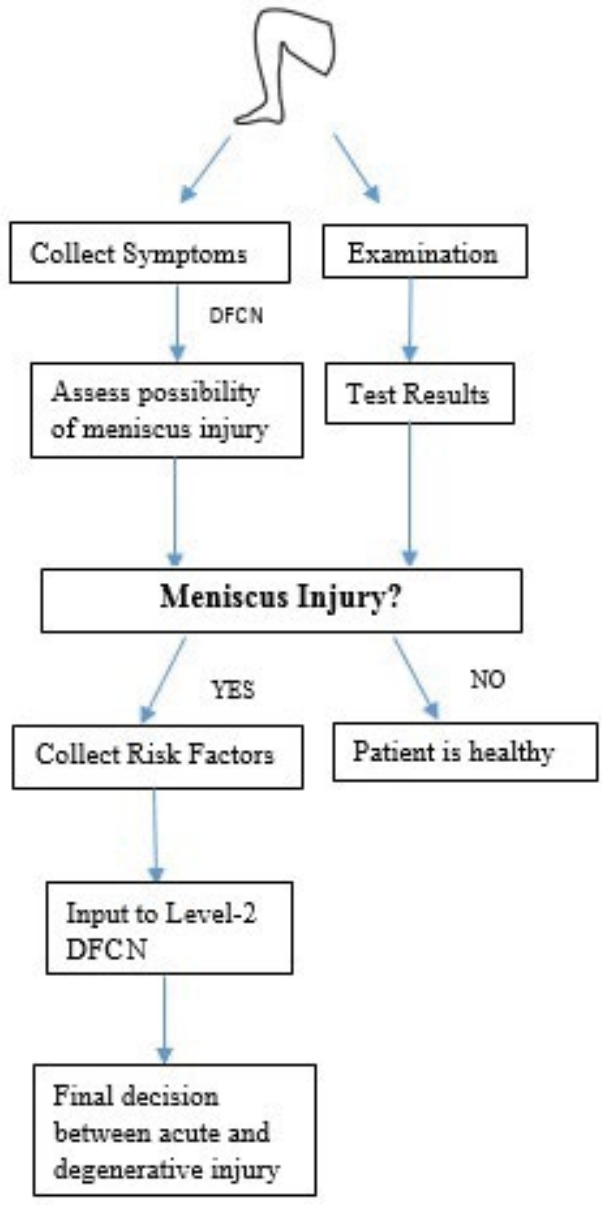

Figure 1: Integrated diagnostic procedure.

Step 3: Physicians construct weight matrices with the interrelationships between Outputs-States, Outputs-Inputs, StatesStates, States-Inputs, using linguistic variables (zero, low, medium, high, very high).

Step 4: Engineers convert weight matrices into numeric values using defuzzification method of Center of Area (CoA).

Step 5: Detect initial states of each patient in time unit " $k$ " that arrived at the hospital.

Step 6: Compute Output in "k" from Eq. (2)-First Level Diagnosis.

Step 7: If patient is positive to meniscus continue to the second level-accurate diagnosis.

\section{Step 8: Collect Risk Factors.}

Step 9: Compute Output in " $k$ " from Eq. (2)-Second Level Diagnosis.

This procedure will be the background of the new platform KneeD. KneeD fully respected the physicians requirements for an easy to use application that all nursing staff and doctors could use as a consultative tool, without any special training. Its functionality won't be limited to an advisory tool, but it will also have a main way of organizing and recording patients' history through a database. The greatest challenge was to create a user-friendly, easy-to-use interface. The exact design was achieved using Net Beans IDE 8.1.

The welcome home page contains the application name as well as the user credentials for the connection as in Figure 2. After logging in, the main menu is displayed with all available options as shown in Figure 3. These are:

Add new patient's data or process someone's existing data with the Add Patient button and store them in the database,

Search based on any patient data is available in the database with the "Search Patient".

Display of all data in tabular form with "Records".

Diagnosis of knee injuries with the "Diagnosis" button and storage in database.

Figure 4 illustrates the details that should be completed by the physician. These are: Name, Sex, Birthday, Social Security Number, Mobile Number, E-mail, Mechanisms of Injury (in checklist format), Symptoms (in checklist format), Risk Factors (in checklist format), Positive Clinical Examination Results (in checklist format), MRI images, Additional Notes.

The functionalities of the screen of Figure 4 are the following:

Save all data and MRI to database and simultaneously store them to the table columns at the top of the same page.

Click any element in the table and move its elements to the blank fields to make changes. Any of these data can be renewed with the "Update" button or completely deleted from the database with the

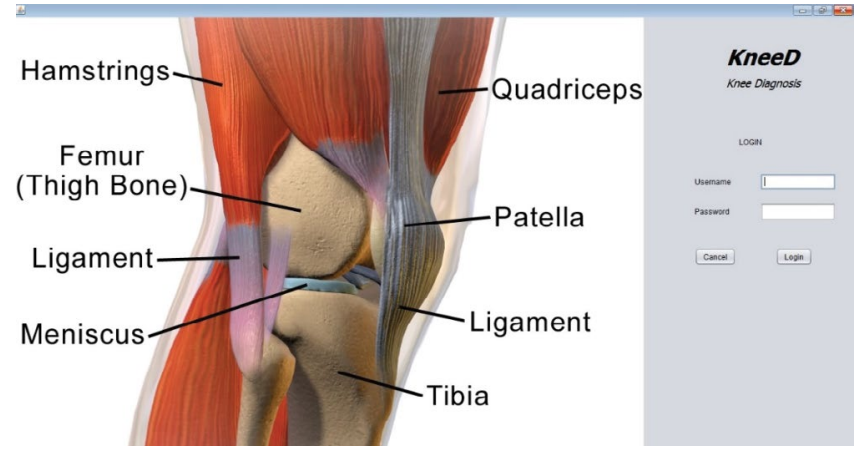

Figure 2: The startup screen.

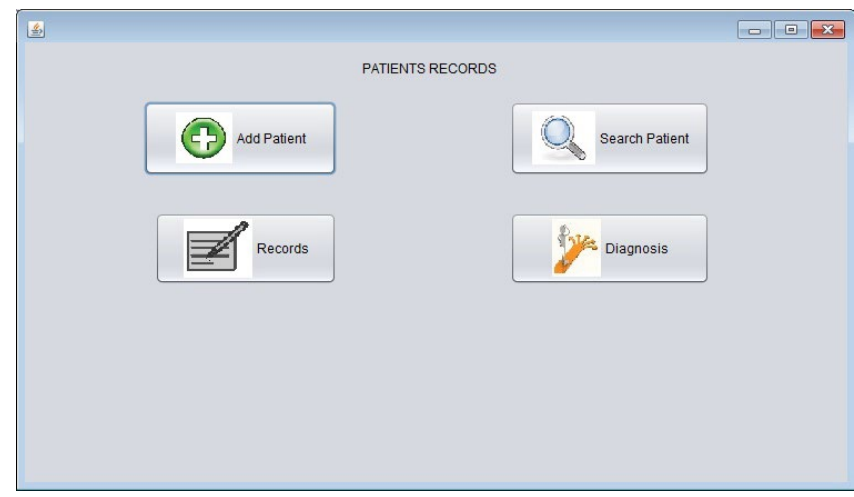

Figure 3: Main menu. 
Citation: Anninou AP, Poulios P, Groumpos PP, Gliatis I (2018) A Novel Software Tool for Detection of Meniscus Injury using Dynamic Fuzzy Cognitive Networks. Physiother Rehabil 3: 155. doi: 10.4172/2573-0312.1000155

Page 4 of 5

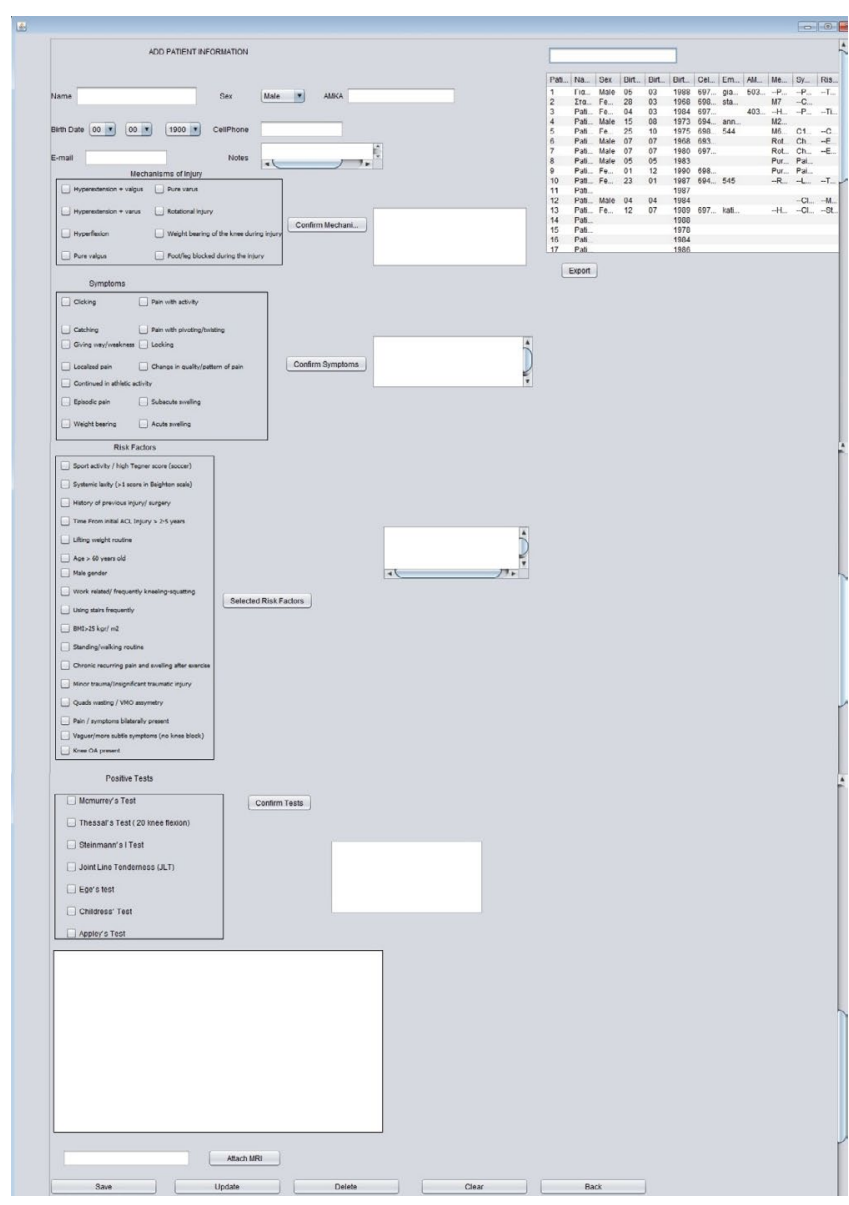

Figure 4: Add data screen.

\section{"Delete" button.}

Clean all cells at any time with "Clear".

Return to main menu can be done with "Back".

Search for any patient based on name.

Export all components to an excel file created and stored in a folder on the desktop named Patients.xlsx.

The "Export" button is responsible for this function. The "Diagnosis" button opens a new screen, in which the patient must be selected to be diagnosed by its name (Figure 5). By selecting the patient and pressing the "Diagnose" button we are leading to a new window with the result, which could be saved in column 15 of the database.

If patient is positive to meniscus injury, we proceed to the second level accurate diagnosis with the corresponding button "Accurate Diagnosis". The possible answers are in Figure 6.

\section{Results}

This is a new pilot model for diagnosing meniscus tear and the differentiation between acute and degenerative injury. The whole procedure from the time that damage happened to patient, to the point that final diagnosis must be decided is being simulated. All simulation results arose from real patients and MRI outcomes evaluated the decisions. The advantages of this two level method are the following:
Modeling the way that the physician makes the examination.

Exploiting experts' knowledge.

17 patients, of which 11 men and 6 women, were collected and diagnosed by KneeD Platform. Their ages range from 18 to 63 years old.

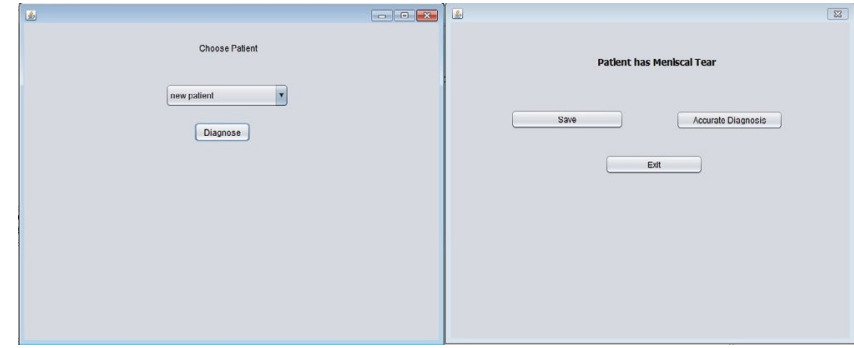

Figure 5: Patient selection and diagnosis.

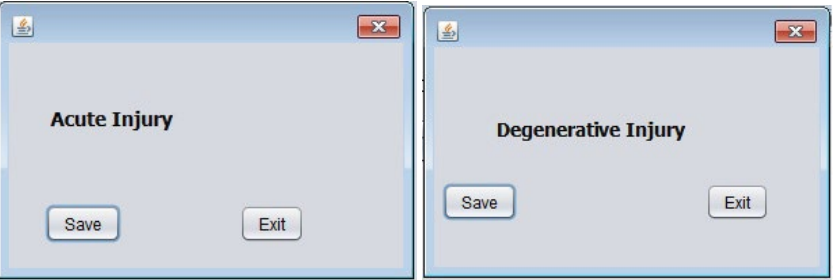

Figure 6: Accurate diagnosis

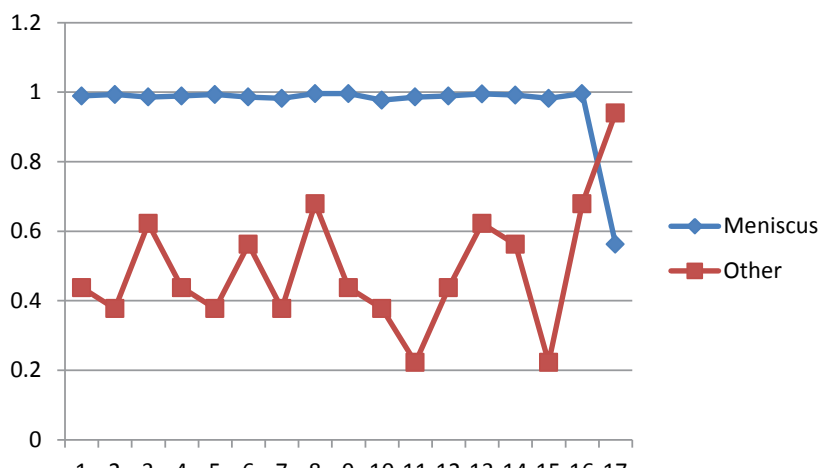

Figure 7: First level outputs.

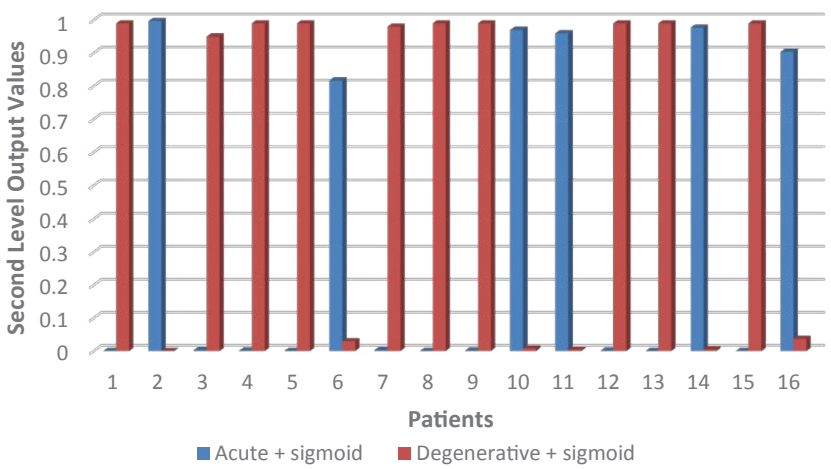

Figure 8: Second level outputs. 
They didn't have prior surgery on their index knee. In addition before the examination, patients had to be relaxed and concentrated in order to contribute to a more accurate assessment with a pillow placed under the knee, keeping it in slight flexion in order to relax the hamstrings. Patients were informed about the study, but all information was provided anonymously without any identifier. That's the reason why no ethical document is shared. The first level numeric diagnoses are illustrated in Figure 7.

Due to the fact that the first level diagnoses are competitive between each other, we observe that only one diagnosis will be the dominant one. Diagnoses with their maximum numeric value being close to 1 consist our final decision. Therefore, in the examined sample, 16 out of 17 patients have meniscal injury, of which 6 have an acute trauma and the rest 10 degenerative injury, as shown in Figure 8. This was ascertained by observing the values close to 1 . These are the dominant decisions, since the alternative choice is too close to 0 because of the competitive nature of the two diagnoses.

These results were tested for their diagnostic accuracy with the following measures: sensitivity and specificity and they were compared with MRI outcomes. The fact that they were in total agreement with each other, concludes to a percentage of $100 \%$ for both sensitivity and specificity. Though this conclusion is very encouraging we need a larger sample of over 60 patients in order to confirm model accuracy.

\section{Conclusions and Future Research}

This article addressed a basic and fundamental issue of modeling meniscus injury using Dynamic Fuzzy Cognitive Networks. This method was used in [10] and was tested in 17 patients. For the first time this specific knee injury modeled in such a simple and comprehensive way. The obtained results have been evaluated by orthopedic surgeons who found them very satisfactory. The method was fast, simple, reliable and cost effective comparing with MRI cost. Modeling in that way helps physicians having a first and accurate diagnosis. However, it is a preliminary study and we need to increase the patient sample in order to evaluate model's reliability and validity.

A very promising research area unveils throughout this paper with a rich future research. This will include simulations with new and more clinical data in order to present new research results and ensure system's validity. The ultimate future goal is the usage of this tool clinically, in real-time medical diagnosis.

\section{References}

1. Noyes F (2009) Noyes' knee disorders, Philadelphia, PA: Saunders Elsevier.

2. Scott WN (2011) Insall \& Scott surgery of the knee. Churchill Livingstone.

3. Judith TE, Ronald KL, Ernest R (1992) Emergency medicine: A comprehensive study guide. Adv Emergen Nursin J 14: 74

4. Morelli V, Bright C, Fields A (2013) Ligamentous injuries of the knee: anterio cruciate, medial collateral, posterior cruciate, and posterolateral corner injuries. Prim Care 40: 335-356

5. Fanelli G (2013) The multiple ligament injured knee: A practical guide to management. (2ndedn), Springer science \& business media.

6. Kocabey Y, Tetik O, Isbell WM, Atay ÖA, Johnson DL (2004) The value of clinical examination versus magnetic resonance imaging in the diagnosis of meniscal tears and anterior cruciate ligament rupture. Arthroscopy 20: 696-700.

7. De Smet AA, Nathan DH, Graf BK, Haaland BA, Fine JP (2008) Clinical and MRI findings associated with false-positive knee MR diagnoses of medial meniscal tears. Am J Roentgenol 191: 93-99.

8. Navali AM, Bazavar M, Mohseni MA, Safari B, Tabrizi A (2013) Arthroscopic evaluation of the accuracy of clinical examination versus MRI in diagnosing meniscus tears and cruciate ligament ruptures. Arch Iran Med 16: 229-232.

9. Kocabey Y, Tetik O, Isbell WM, Atay ÖA, Johnson DL (2004). The value of clinical examination versus magnetic resonance imaging in the diagnosis of meniscal tears and anterior cruciate ligament rupture. Arthroscopy 20: 696-700.

10. Anninou AP, Groumpos PP, Poulios P, Gkliatis I (2017) A New approach of dynamic fuzzy cognitive knowledge networks in modelling diagnosing process of meniscus injury. IFAC-papers online 50: 5861-5866.

11. Anninou PA, Groumpos PP (2014) Modeling of parkinson's disease using fuzzy cognitive maps and non-linear hebbian learning. International J Artifi Intellig Tools 23: 17.

12. Kosko B (1986) Fuzzy cognitive maps. Internat J Man-Mach Stud 24: 65-75.

13. Saade JJ (1996) An unifying approach to defuzzification and comparison of the outputs of fuzzy controllers. IEEE Transac Fuzzy System 4: 227-237.

14. Stylios CD, Groumpos PP (1998) The challenge of modelling supervisory systems using fuzzy cognitive maps. J Intellig Manufact 9: 339. 